\title{
Simulative Analysis on Hybrid Optical Amplifier in Fiber Distributed Ring for WAN
}

\author{
Jaspreet Kaur \\ M-Tech Scholar \\ CSE Department \\ DAVIET,Jalandhar
}

\author{
Rajesh Kochher \\ Assistant Prof. \\ IT Department \\ DAVIET,Jalandhar
}

\begin{abstract}
The tremendous growth of the high speed Internet and data traffic has created an enormous demand for transmission bandwidth of dense wavelength division multiplexed (DWDM) optical communication systems. With the advancement of wideband fiber-optical amplifiers, DWDM optical transmission systems are capable of providing capacities in excess of hundreds of gigabits per second over hundreds of kilometers on a single pair of fibres in long-haul networks. In this paper the investigations in FIBRE Distributed ring for wide area network for six nodes, 45 channels. The main target of this research work is to reduce the data impairment in the data transmission. In this manuscript we investigate the performance on the basis of $\mathrm{Q}$ factor, Eye diagram and BER from the simulation setup designed on Hybrid Optical Amplifier in Fiber Distributed Ring for Wide Area Network.
\end{abstract}

\section{Keywords}

FDDI,SOA,RAMAN, EDFA, WDM, HA.

\section{INTRODUCTION}

Due to increase in bandwidth demand in the network system the use of optical fiber is recommended. To achieve the higher data transfer rate to support new multimedia application and services different network providers are moving towards optical network. To increase optical network capacity the WDM system is emerged in optical communication system. The WDM system transmit multiple channels over a single fiber [24]. The tremendous growth of the high speed Internet and

data traffic has created an enormous demand for transmission bandwidth of dense wavelength division multiplexed (DWDM) optical communication systems [18].To counter attenuation and dispersion effect and to enhance the performance of the DWDM various optical amplifier are needed. In DWDM system, it is desirable to set a very narrow grid of optical carriers in order to allow more channels in the same optical bandwidth. DWDM systems involve high capacity long haul transmission. Hybrid Amplifiers (HA) are an enabling and promising technology for future DWDM. Besides the bandwidth and noise level issues, HAs are designed in order to maximize the fiber span and/or to minimize the impairments of fiber non-linearities.

\section{LITERATURE REVIEW}

Priya Dahiya, Himanshi Saini, Amit Kumar Garg[2014][8] In this paper several optical amplifiers are discussed with their application that are suitable for the low cost, high performance applications of DWDM systems. Their advantages are integrated to improve the performance of an optical communication system. Hybrid optical amplifiers are useful to combine the benefits and compensate for the drawbacks of different optical amplifiers for enhancement of optical networks is greater than that of fibre distributed ring and delay experienced by theEthernet. Olusegun O. Omitola, Segun O.Olatinwo and O.Shoewu [2014][17] In this paper, the authors investigate and evaluate the behavior of throughput and delay on twodifferent technology: Ethernet and FIBER DISTRIBUTED RING. They make two scenarios with 20 nodes and designthem with OPNET-MODELER simulator, bothtechnologies operate at a speed of 100Mbps. Theyconclude that throughput in Ethernet network is more thanthat of FIBER DISTRIBUTED RING, while using the same number of nodes. Raj Jain[1990][2]In this paper, author described that the ANSI developed the 100 mbp LAN which used the timed token access method and allow various station connected with total fiber length of $200 \mathrm{~km}$. The performance metrics of response time, efficiency and maximum access delay are considered, where efficiency define the ratio of maximum obtainable throughput to the nominal bandwidth of the network and access delay is defined as the time it takes to receive a usable token. The performance of FIBER DISTRIBUTED RING depend on some workload parameters like the arrival pattern, frame size and configuration parameters such as number of station on the ring, extent of the ring, and number of station that are waiting to transmit. The performance is also affected by a parameter Target Token Rotation Time (TTRT), which is controlled by network manager. Rahul Jashvantbhai Pandya, Vinod Chandra, and Devi Chadha[2014][3] In this paper the author described the power economy (PE) and impairment awareness (IA) which are crucial issue in optical network. Transparency is needed to achieve power economy in optical networks, as it is required to avoid the regeneration of an optical signals. This leads to an accumulation of noise due to the physical layer impairments which result in loss of quality of the signal. They have optimized the selective usage of traffic grooming, mixed regeneration and all the optical wavelength conversion using integer linear programming to increase the $\mathrm{PE}$ and IA simultaneously. Sercote N. Abdullah, Hussein A. Jawad and Hussam A. Mohammed [2002][7] In this paper the author concluded that the maximum throughput and maximum access delay are directly proportional to length of the transmitted information. A trade off between the transmitted information length and the maximum access delay is needed.

Regis S. Fan and R. Brian Hooker[2000][10] In this paper, they investigate that the use of Semiconductor optical amplifiers with passive polymer waveguides to make hybrid switches of varying sizes. The optical amplifiers serve dual purposes, getting the signal and amplifying the signal. Amplification is needed in order to offset the losses associated with the passive waveguide elements as well as the losses from component misalignments in the switch module. They find that the largest switch module siz can be made with the architecture used. They also calculate the maximum number of switch modules which can be cascaded in order to retain a bit-error rate. 


\section{RESULTS}

To overcome the problems of attenuation and dispersion effect of DWDM systems in distributed ring architecture.We apply hybrid optical amplifiers. To gain more accurate results regarding the loss. We design a simulation scenario of hybrid optical amplifier in fibre distributed ring network on wide area network. In this design we connect various nodes using optical fibre cable on every node. We apply the combination of various optical hybrid amplifier and choose the best one form other. The Design is valid upto 45 channel connected to a node with the help of add drop multiplier add or drop various node in the design.

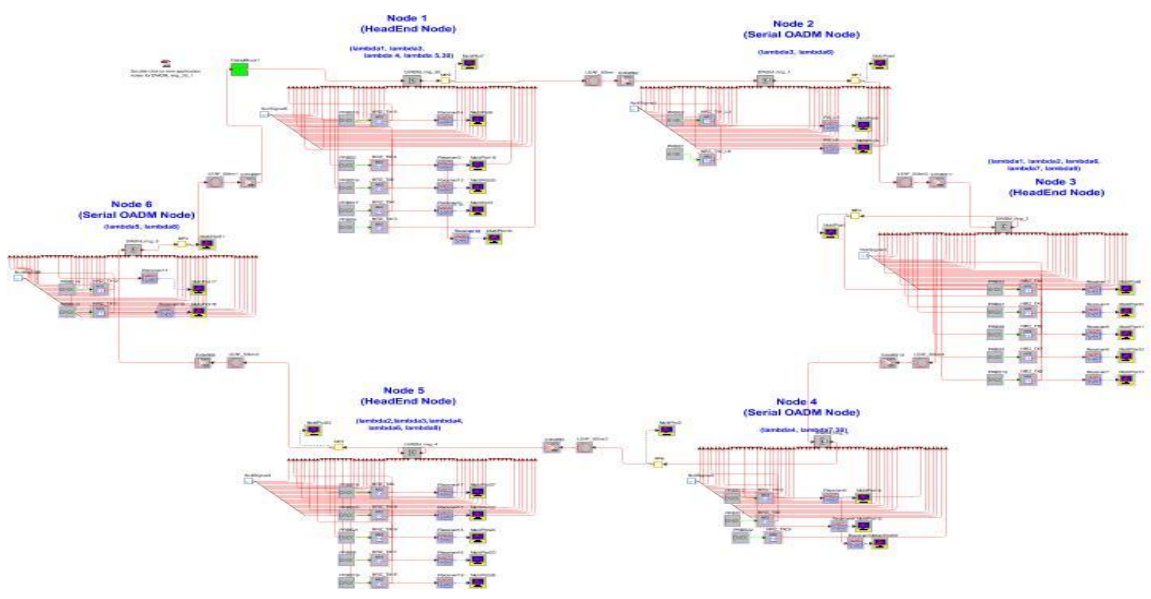

Figure1. The Design simulation scenario of Hybrid Optical Amplifier in Fiber Distributed Ring Network on Wide Area Network.

When Input is coming to a particular node it goes to the Electrical generator which convert the Electrical data into optical data, that data goes to the External modulator in which the data is modulated finally the optical normalize normalize the data after that the output is generate.On Applying various hybrid Amplifier on this design we check out the performance of FIBRE Distributed ring architecture for wide area network on the basis of eye diagram, BER and Q factor.
Here eye diagram determines how sensitive the signal is to timing error. More open the eye more better the transmission can occur. It has been observed that the eye diagram of the system changes for every channel of a node. It has been observed that the bit error rate increases and the chances of cross talk and data loose is also increased due to increase the distance. The eye is more open in EDFA and SOA combination.

Table 1. Results of Eye diagram parameter.

\begin{tabular}{|c|c|c|c|}
\hline Node 1 & EDFA+ RAMAN Amplifier & EDFA+SOA Amplifier & EDFA+SOA+RAMAN Amplifier \\
\hline channel3 & Iime (s) & IstmpMutililot18 Eye Dlagram & $\varepsilon_{0}^{0.03}$ \\
\hline Channel 4 & IstmpMultiplot20 Eye Diagram & Time (s) & IstmpMultiPlot12 Eye Diagram \\
\hline
\end{tabular}




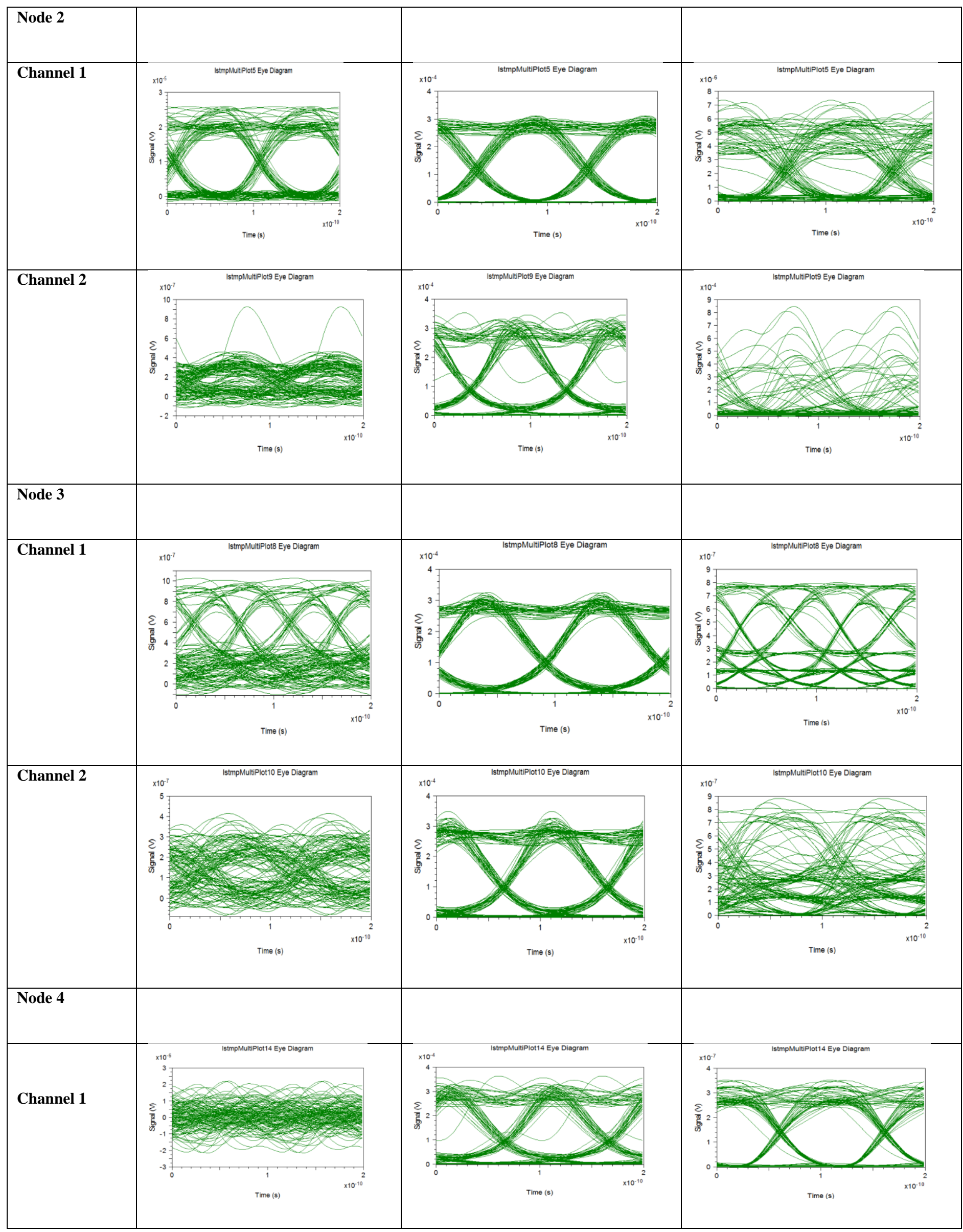




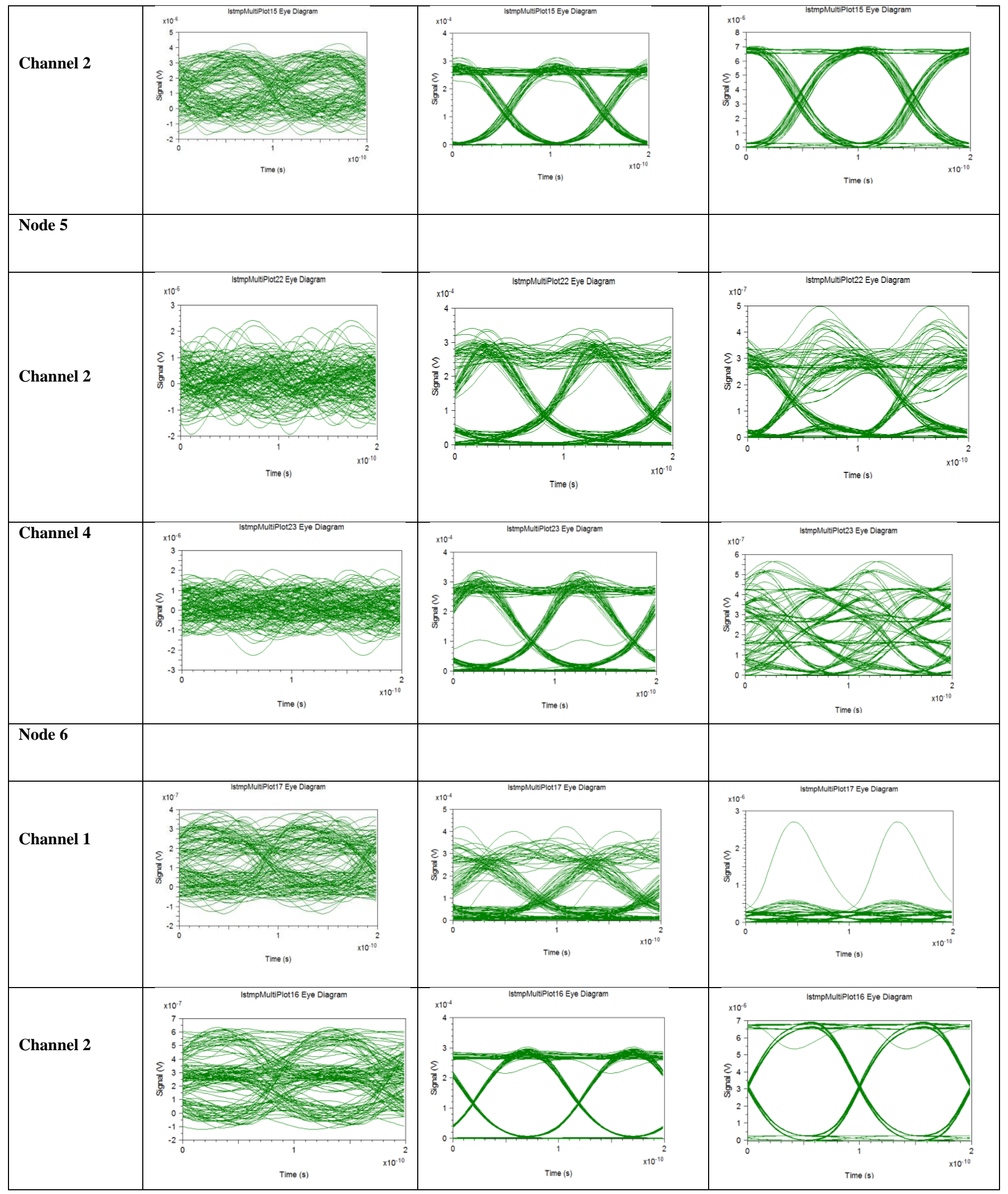

Next parameter is BER that is bit error rate. It represent the error occurred in the transmission of data. We always try to minimize the ber to improve the data transmission speed. Combination of EDFA and SOA provide less ber value. 
Table 2. Results of BER parameter.

\begin{tabular}{|l|l|l|l|}
\hline Node 1 & EDFA+ RAMAN Amplifier & EDFA+SOA Amplifier & EDFA+SOA+RAMAN Amplifier \\
\hline Channel 3 & $2.3265 \mathrm{e}-002$ & $3.1388 \mathrm{e}-024$ & $3.12 \mathrm{e}-2$ \\
\hline Channel 4 & $2.3265 \mathrm{e}-002$ & $4.1084 \mathrm{e}-017$ & $2.15 \mathrm{e}-3$ \\
\hline Node 2 & & & \\
\hline Channel 1 & $4.1555 \mathrm{e}-013$ & $3.4716 \mathrm{e}-044$ & $3.93 \mathrm{e}-6$ \\
\hline Channel 2 & $1.570 \mathrm{e}-02$ & $1.3242 \mathrm{e}-011$ & $1.2 \mathrm{e}-1$ \\
\hline Node 3 & & & \\
\hline Channel 1 & $1.0000 \mathrm{e}+000$ & $4.4117 \mathrm{e}-030$ & $2.51 \mathrm{e}-4$ \\
\hline Channel 2 & $1.0000 \mathrm{e}+000$ & $4.9751 \mathrm{e}-020$ & $5.2 \mathrm{e}-2$ \\
\hline Node 4 & & & \\
\hline Channel 1 & $1.0000 \mathrm{e}+000$ & $5.3878 \mathrm{e}-011$ & $2.6 \mathrm{e}-4$ \\
\hline Channel 2 & $1.5048 \mathrm{e}-002$ & $1.7128 \mathrm{e}-052$ & $5.2 \mathrm{e}-12$ \\
\hline Node 5 & & & $1.0698 \mathrm{e}-095$ \\
\hline Channel 2 & $1.0000 \mathrm{e}+000$ & $4.5441 \mathrm{e}-017$ & $5.2 \mathrm{e}-12$ \\
\hline Channel 4 & $1.0000 \mathrm{e}+000$ & & $1.0000 \mathrm{e}-01$ \\
\hline Node 6 & & $1.1146 \mathrm{e}-003$ & \\
\hline Channel 1 & $1.0000 \mathrm{e}+000$ & $1.0000 \mathrm{e}+000$ \\
\hline Channel 2 & $1.0000 \mathrm{e}+000$ & & $2.56 \mathrm{e}-15$ \\
\hline
\end{tabular}

Last parameter is Q-factor. High Q-factor is required for to minimize the ber factor and maximize the Q-factor. better transmission. For better transmission we always try

Table 3. Results of Q-factor parameter

\begin{tabular}{|l|l|l|l|}
\hline Node 1 & $\begin{array}{l}\text { EDFA+ RAMAN } \\
\text { Amplifier }\end{array}$ & EDFA+SOA Amplifier & EDFA+SOA+RAMAN Amplifier \\
\hline Channel 3 & 3.8 & 22.6 & 1.36 \\
\hline Channel 4 & 3.8 & 16.4 & 1.87 \\
\hline Node 2 & & & 5.34 \\
\hline Channel 1 & 12.3 & 36.2 & .83 \\
\hline Channel 2 & 3 & 9.2 & \\
\hline Node 3 & & & 5.29 \\
\hline Channel 1 & 0 & 24.6 & 1.51 \\
\hline Channel 2 & 0 & 18.3 & \\
\hline Node 4 & & & 3.8 \\
\hline Channel 1 & .56 & 10.5 & 10.73 \\
\hline Channel 2 & 3.1 & 47.8 & 13.72 \\
\hline Node 5 & & 16.9 & 0.92 \\
\hline Channel 2 & 0.34 & 16 & \\
\hline Channel 4 & 0.38 & & \\
\hline Node 6 & & 67.3 & \\
\hline Channel 1 & 1.32 & & \\
\hline Channel 2 & .67 & & \\
\hline
\end{tabular}




\section{CONCLUSION AND FUTURE SCOPE}

On applying various hybrid optical amplifiers in fibre distributed ring for wide area network we conclude that the various impairment issues are minimized and fibre span is maximized in SOA and EDFA Combination. Further the optimized performance can be obtained by investigating the fibre distributed ring for wide area network by different modulation format like RZ and CRZ on the bases of Q factor, eye diagram, \&BER. Further,in future performance of Hybrid optical in Fiber Distributed Ring for Wide Area Network is improved using different Modulation formats.

\section{REFERENCES}

[1] Henry S. Yang, Barry A. Spinney, and Stephen Towning, “"FDDI Data Link Development" publication year 1991, Vol. 3 No. 2 Spring, Digital Technical Journal.

[2] Raj Jain, “ Performance Analysis of FDDI Token Ring Networks:Effect of Parameters and Guidelines for Setting TTRT" publication year 1990, JainOErlang.DEC.Com

[3] Rahul Jashvantbhai Pandya, Vinod Chandra, and Devi Chadha, "Simultaneous Optimization of Power Economy and Impairment Awareness by Traffic Grooming,Mixed Regeneration, and All Optical Wavelength Conversion With an Experimental Demonstration" publication year:2014, VOL. 32, NO. 24, JOURNAL OF LIGHTWAVE TECHNOLOGY.

[4] Matteo Fiorani, Slavisa Aleksic, Paolo Monti, Jiajia Chen,Maurizio Casoni, and Lena Wosinska, "Energy Efficiency of an Integrated Intra-Data-Center and Core Network With Edge Caching" publication year 2014,VOL. 6, NO. 4,J. OPT. COMMUN. NETW.

[5] S. Peiris, N. Madamopoulos, N. Antoniades, D. Richards, M. A. Ummy, and R. Dorsinville, "Engineering an Extended Gain Bandwidth Hybrid Raman-Optical Parametric Amplifier for Next Generation CWDM PON" publication year: 2014, VOL. 32, NO. 5, JOURNAL OF LIGHTWAVE TECHNOLOGY.

[6] Takaya Miyazawa, Member, IEEE, Member, OSA, and Naoya Wada, Member, IEEE, "Optical Packet and Circuit Integrated Networks and Software Defined Networking Extension",publication year2014, VOL.32, NO.16, JOURNAL OF LIGHTWAVE TECHNOLOGY.

[7] Sercote N. Abdullah ,Hussein A. Jawad and Hussam A. Mohammed, "Design Considerations of Laser Source in a Ring Network Based on Fiber Distributed Data Interface (FDDI)" Publication year:2002, Vol. 1, No. 1.

[8] Priya Dahiya, Himanshi Saini and Amit Kumar Garg, "Impact of Hybrid Optical Amplifiers in High Speed Networks Publication Year:2014,VOL.3,No.3,International Journal of wireless Communication and Networking Technologies.

[9] Shingo Kawai, Hiroji Masuda, IEEE, Ken-Ichi Suzuki and Kazuo Aida, "Wide-Bandwidth and Long-Distance WDM Transmission Using Highly Gain-Flattened Hybrid Amplifier " Publication year:1999, , VOL. 11, NO. 7, IEEE PHOTONICS TECHNOLOGY LETTERS.

[10] Regis S. Fan and R. Brian Hooker, "Hybrid Optical Switch Using Passive PolymerWaveguides and
Semiconductor Optical Amplifiers", Publication Year:2000, VOL. 18, NO. 4,JOURNAL OF LIGHTWAVE TEHCNOLOGY.

[11] Hyuk-Choon Kwon, Yong-Yuk Won, and Sang-Kook Han, "Noise Suppressed Fabry-Pérot Laser Diode With Gain-Saturated Semiconductor Optical Amplifier for Hybrid WDM/SCM-PON Link ", Publication Year:2006, , VOL. 18, NO. 4, IEEE PHOTONICS TECHNOLOGY LETTERS.

[12] Tadashi Sakamoto, Shin-ichi Aozasa, Makoto Yamada, and Makoto Shimizu, "Hybrid Fiber Amplifiers Consisting of Cascaded TDFA and EDFA for WDM Signals",Publication Year:2006, VOL. 24, NO. 6, JOURNAL OF LIGHTWAVE TECHNOLOGY.

[13] Scott Schube and Marco Mazzini, "Testing and Interoperability of 10GBASE-LRM Optical Interfaces",Publication Year:2007,IEEE.

[14] P. P. Iannone, H. H. Lee, K. C. Reichmann, X. Zhou, M. Du, B. Pálsdóttir, K. Feder,P. Westbrook, K. Brar, J. Mann, and L. Spiekman, "Four Extended-Reach TDM PONs Sharing a Bidirectional Hybrid CWDM Amplifier "Publication Year:2008, , VOL. 26, NO. 1, JOURNAL OF LIGHTWAVE TECHNOLOGY.

[15] Amin M. E.-A. Diab, Jonathan D. Ingham,Richard V. Penty, and Ian H. White, "10-Gb/s Transmission on Single-Wavelength Multichannel SCM-Based FDDIGrade MMFLinks at Lengths Over 300 m: A Statistical Investigation",Publication Year:2007,VOL.25,NO.10, IEEE.

[16] Minwan Jung, You Min Chang, and Ju Han Lee, “ A Band-Separated, Bidirectional Amplifier Based on Erbium-Doped Bismuth Fiber for Long-Reach Hybrid DWDM-TDM Passive Optical Networks" Publication Year: 2012,VOL. 4, NO. 3,Jou. OPT. COMMUN. NETW

[17] Olusegun O. Omitola,Segun O. Olatinwo and O. Shoewu, "Evaluation and Investigation of Throughput and Delay on Ethernet and FDDI Technologies using OPNET ", Publication year 2014,VOL. 15,NO.1,Spring.

[18] Bárbara Dumas and Ricardo Olivares, "Transient Link Control Technique Appliedto Optical Hybrid Amplifier (EDFÅ̊DFRA) Cascades", Publication Year :2012, VOL.4,NO.11,Journal optical communication network.

[19] Simranjit Singh and R. S. Kaler, "Flat-Gain L-Band Raman-EDFA Hybrid Optical Amplifier for Dense Wavelength Division Multiplexed System", Publication Year :2013, VOL. 25, NO. 3,IEEE PHOTONICS TECHNOLOGY LETTERS.

[20] Stanley Cheung, Yasumasa Kawakita, Kuanping Shang, and S. J. Ben Yoo, "Theory and Design Optimization of Energy-Efficient Hydrophobic Wafer-bonded III-V/Si HybridSemiconductor Optical Amplifiers", Publication Year 2013, VOL. 31, NO. 24,JOURNAL OF LIGHTWAVE TECHNOLOGY.

[21] Simranjit Singh and R. S. Kaler, "Novel Optical FlatGain Hybrid Amplifier for Dense Wavelength Division Multiplexed System",Publication Year 2014,VOL.26,NO.2, IEEE PHOTONICS TECHNOLOGY LETTERS. 
[22] Li Tao, Yu Ji, Jie Liu, Alan Pak Tao Lau, Nan Chi, and Chao $\mathrm{Lu}$, "Advanced Modulation Formats for Short Reach Optical Communication Systems",

[23] Ishfan Vakil and James M. Conrad, "Design of a Data CommunicationsHubforuseinResearchandEducation"Pub licaton Year:2006,IEEE
[24] Pierre Dersin, "Availability of Data Communication Networks in Automated UrbanTrain Systems",Publication Year: 2014,IEEE.

[25] Chi-Hao Cheng, "Signal Processing for Optical Communication", Publication Year:2006, IEEE.

[26] Raj Jain, "FDDI:Current issues and future plans",publication ”,publication year:1993, IEEE. 